\title{
A Compact Graphene Based Nano-Antenna for Communication in Nano-Network
}

\author{
M. Ram Kumar,** \\ ${ }^{1}$ Department of Computer Science and Engineering, Thirumalai Engineering College, Kanchipuram, Tamilnadu, and \\ India \\ Email: mramkumar0087@gmail.com \\ *Corresponding Author: M. Ram Kumar, Email: mramkumar0087@gmail.com
}

How to cite this paper: M. Ram Kumar (2019) A Compact Graphene Based Nano-Antenna For Communication in Nano-Network. Journal of the Institute of Electronics and Computer, 1, 17-27. https://doi.org/10.33969/JIEC.2019.11003.

Received: November 14, 2019

Accepted: November 19, 2019

Published: November 19, 2019

Copyright $(2020$ by author(s) and Institute of Electronics and Computer.

This work is licensed under the Creative Commons Attribution International License (CC BY 4.0).

http://creativecommons.org/licenses/by/4.0/

cc) (i) Open Access

\begin{abstract}
Due to recent advances in nanotechnology, the use of nano-devices and its network becomes more popular in the field of medical, commercial and military applications. One of the major issues in designing nano-network is miniaturization of nano-devices which are limited due to communication antenna used in that device and its power constraints. At $1000 \mathrm{~nm}$ size, an antenna resonates at around $100 \mathrm{THz}$ which suffers from greater propagation loss and provides signal coverage of micrometer distances. Hence there is a need for nano-antenna with reduced size and also operating at mid infrared frequencies to provide a good signal coverage. In this paper, Graphene-based nano-antenna is presented. The model resonates at $55 \mathrm{THz}$ frequency with a peak gain of $5.47 \mathrm{~dB}$ in the propagation direction. The model exploits the principle of surface plasma polariton waves for miniaturization and achieves $50 \%$ size reduction when compared to conventional nano-antenna and best suitable for nano-network communications.
\end{abstract}

\section{Keywords}

Nano Antenna; Radiation Pattern; Reflection Coefficient; Microstrip Antenna; Surface Plasma Polariton.

\section{Introduction}

Microstrip antenna plays an important role in the field of wireless communications due to its compact size, light weight and better ease of fabrication [1]-[2]. In last few decades, the nano-devices play a vital role in the fields of biomedical and military applications. Designing of antennas suitable for these nano-devices is a big challenge due to its size and power constraints. Evaluation of nano-antenna at the mid-infrared frequency is carried in [3]. With limited availability of power, it is 
impossible to establish communication in nano-networks using materials like copper etc. This can be mitigated by using graphene material in place of copper. Because of its electrical property, graphene can act as a good conductive sheet and do work as traditional antenna made of copper. At the size of nano-meters, the antenna made of copper or other metallic materials resonates at very high frequencies (hundreds of terahertz). For example dipole antenna of size 1000nm long resonates at $150 \mathrm{THz}$ which results in increased propagation loss and hence covers small regions. This puts a limitation in using of these antennas in nano-networks. Therefore there is need to design antenna at nano-scale operating at low frequency. This can be achieved by introducing nano-materials especially graphene having one atom thick in miniature antennas [4]. Josep Miquel Jornet and Akyildiz [5], proposed an architecture for communication in nano-networks and its integration with existing communication networks. They modeled and analyzed nano-based antenna and observed that when graphene is excited by means of electromagnetic waves, the electrons in the graphene moves back and forth at low frequencies [6] and are called surface plasma polariton (SPP) and its behavior is studied and analyzed [7]-[9]. Plasmonic antennas have an electrical length greater than its physical length and hence resonating at lower frequencies compared to a conventional antenna having same dimension [10], and hence these antennas are more compact in size and more suitable for nano-devices. 1-D Photonic Crystal (PCs) Cover is used to improve the gain of the antenna [11]. Among many materials, graphene is attracting many researchers in developing nano-antenna due to its unique electrical properties [12]. Renato Iovine et al. [13] studied the electromagnetic behavior of graphene nano-particles which consist of circular and square rings embedded in the dielectric environment. They concluded that a larger bandwidth is obtained by varying the geometrical structure of the nano-particles. Yousif and Samra [14], discussed the influence of antenna length, gap dimensions and shapes on the performance of optical nano-antenna and observed that the efficiency of the antenna is improved by polarization parallel to antenna axis. Seyed Arash Naghdehforushha and Gholamreza Moradi [15], demonstarted Plasmonic patch antenna based on graphene with tunable terahertz band communications. In [16], Wideband $\mathrm{THz}$ antenna using graphene based tunable circular reactive impedance substrate is presented. From the literature discussed above, the effect of graphene layer on the performance of the antenna such as impedance and radiation characteristics is not studied.

In this article, a graphene-based nano-antenna operating at terahertz band is modeled and analyzed by using finite difference time domain (FDTD) method and commercial software. The model exploits the behavior of surface plasma polariton 
in order to achieve size reduction and to operate at lower band frequencies simultaneously. Parametric analysis is carried on the impact of substrate thickness and its permittivity $(\mathcal{E})$ and dimensions of a graphene layer on performances of antenna impedances and its radiation characteristics. The results obtained are compared with a traditional antenna made of metallic material and shows that proposed model achieves good radiation and impedance characteristics with greater reduction in size, having half the size of conventional nano-antenna. Thus the proposed antenna model is suitable for establishing communication in wireless nano-networks.

\section{Principle of Proposed Antenna Design}

\subsection{Surface Plasma Polariton (SPP)}

Surface Plasma Polariton (SPP) waves are generated at the interface between two metals by passing electrons into one of the metals. These waves propagate at much slower speed along the interface and virtually increase the electrical length of the antenna when compared to the physical length of the antenna. Hence they resonate at much lower frequencies. The proposed model exploits this principle to reduce the overall size of the antenna and thereby make it suitable for nano-networks communications.

\subsection{Geometry of Proposed Antenna}

In order to generate SPP waves, a source is needed to inject electrons in the superstrate medium. A standard patch antenna of size $900 \mathrm{~nm} \times 900 \mathrm{~nm} \times 1 \mathrm{~nm}$ is chosen to operate at $100 \mathrm{THz}$ near-infrared frequency and is used for this purpose as shown in Fig. 1(a). This is in conflict with conventional RF antenna design. In conventional dipole antenna designs, the height of the antenna is directly proportional to the operating wavelength which is given by $\mathrm{h} \approx \lambda / 2$.

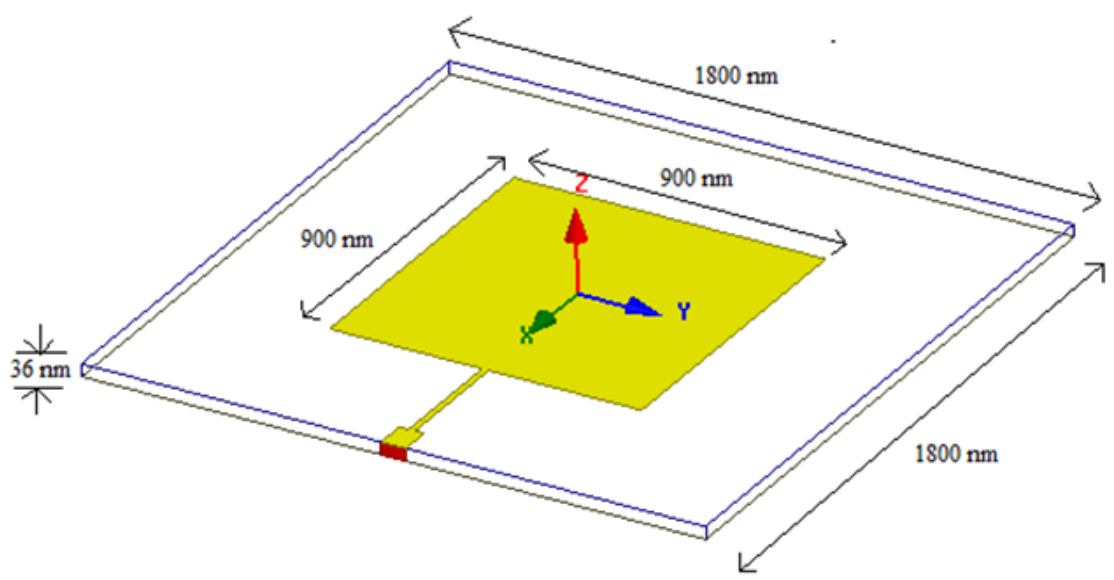

(a) 


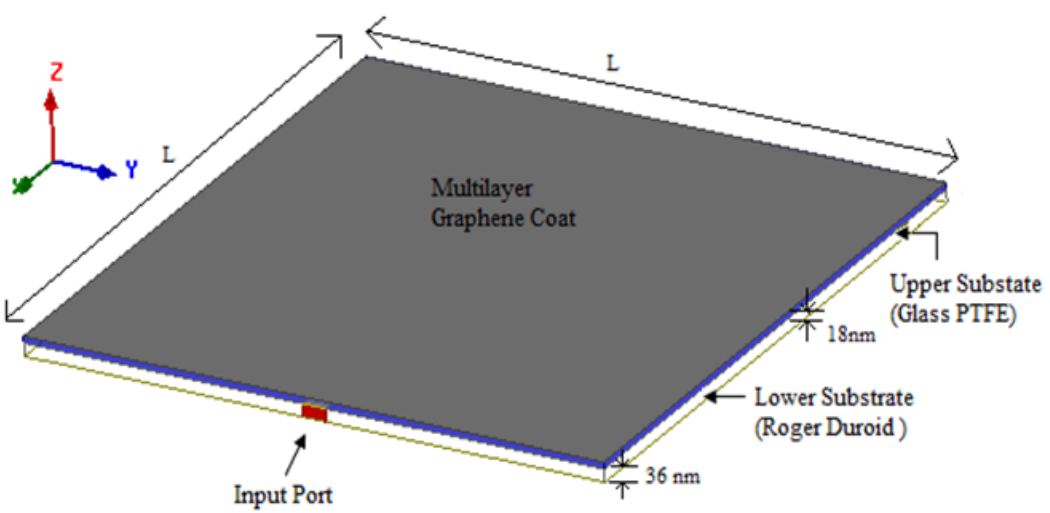

(b)

Figure 1. Geometry of nano-antenna. (a) Dimension of patch antenna which acts a source to excite SPP waves. (b) Final prototype antenna with multilayer graphene.

However, this relation cannot be applied at optical frequencies in nano-antennas since the radiation emitted pierces into the metal and generates free electron oscillations along this surface. Therefore for nano-antennas, the length is not directly related with its wavelength $\lambda$ and hence the dimensions of the nano-antenna depends on a shorter effective wavelength $\lambda_{\text {eff }}<\lambda$ which are dependent on nano-antenna's material properties [17] given below.

$$
\lambda_{e f f}=n_{1}+n_{2}\left[\frac{\lambda}{\lambda_{p}}\right]
$$

Where $\lambda_{\boldsymbol{p}}$ is the plasma wavelength corresponding to metallic material used in nano-antenna and $\mathrm{n} 1$ and $\mathrm{n} 2$ are coefficients depending on nano-antenna material properties. For a single antenna element having radius $\mathrm{R}$ and effective dielectric constant $\varepsilon$, the effective wavelength is given by

$$
\lambda_{e f f}=2 \pi R\left(n_{1}+n_{2} \frac{\lambda}{\lambda_{p}}\right)-4 R
$$

The patch is modeled on roger substrate having a permittivity $(\varepsilon)$ of 2.2 and a thickness of $36 \mathrm{~nm}$. The length and width of roger substrate is taken as $1800 \mathrm{~nm} \mathrm{x}$ 1800nm. Fig. 1(b) shows the geometry of proposed nano-antenna. A glass material having a permittivity $(\mathcal{E})$ of 2.5 is taken as a dielectric medium. Multilayer graphene is coated on the glass dielectric medium.

\section{Parametric Analysis}

The permittivity $(\mathcal{E})$ of the material and the dimension of the graphene (L) layer affects the overall performance of the antenna and hence parametric analysis is carried on these parameters and its effect on antenna performance is studied. 


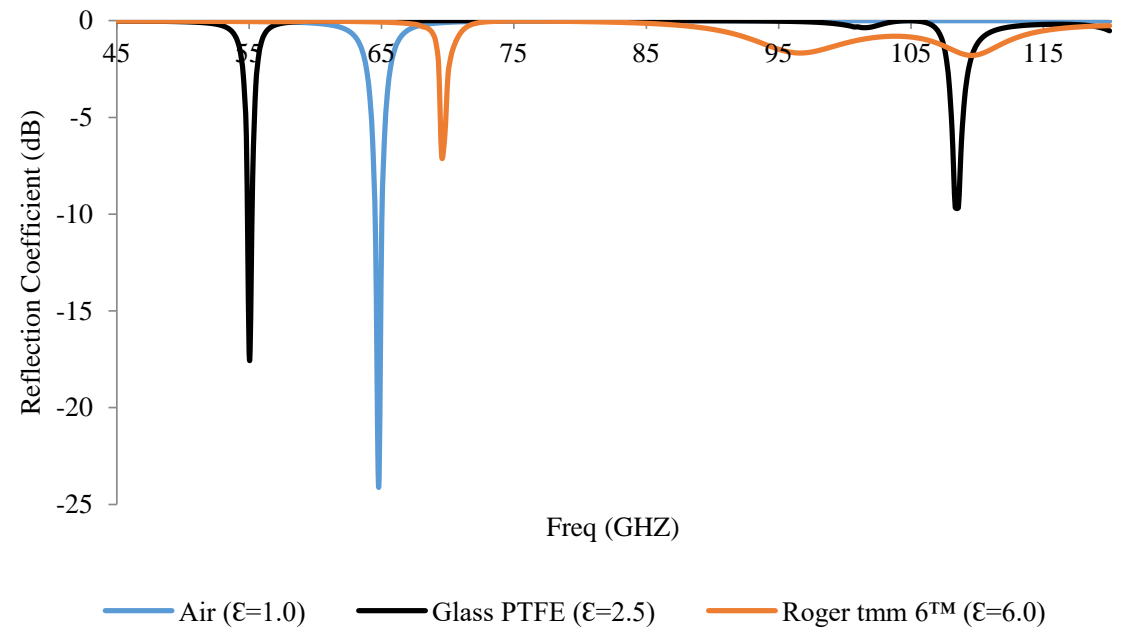

Figure 2. Effect of material permittivity $(\mathcal{E})$ on antenna performance.

Fig. 2 shows effects on changing permittivity $(\varepsilon)$ of the upper substrate on operating frequency of the antenna. Three different dielectric materials having a wide variation in permittivity $(\varepsilon)$ is chosen and its effect on operating frequency is shown. It is observed that glass material performs well when compared to air and roger substrate.

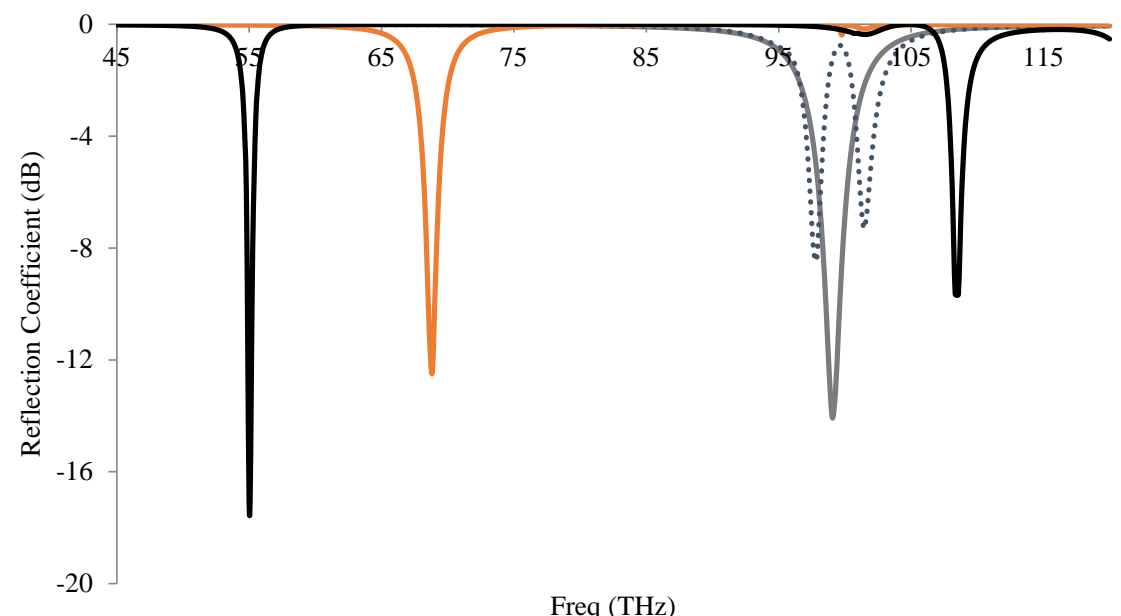

Figure 3. Effect of length (L) of a graphene layer on antenna performance.

Fig. 3 shows the effect of length of the graphene coat on antenna performance. It is observed that with an increase in the length of the graphene coat, the antenna resonates towards the lower band. It is due to the fact that when the graphene 
dimension is lesser than the overall surface dimension of the glass material, the interface between glass and the dielectric material is lesser and hence the SPP propagates for smaller distance resulting in the generation of high-frequency components. Therefore the length and width of graphene layer must cover the entire surface of the dielectric material in order to increase the interface length. This virtually increases the electrical length of the antenna and thereby resonating at lower frequencies which are observed in Fig. 3.

\section{Results and Analysis}

From parametric analysis discussed above, the optimum length of graphene layer is taken as $1800 \mathrm{~nm}$ and effective permittivity $(\mathcal{E})$ of the dielectric material (glass) is taken as 2.5. Fig.4 shows the electric field distribution of multilayer graphene coat upon excitation at the resonant frequency.

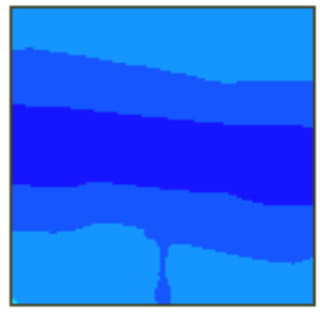

(a) $t=0 \& \mathrm{~T}$

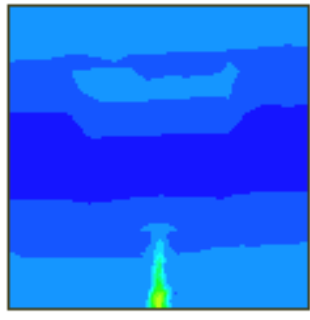

(c) $\mathrm{t}=2 \mathrm{~T} / 4$

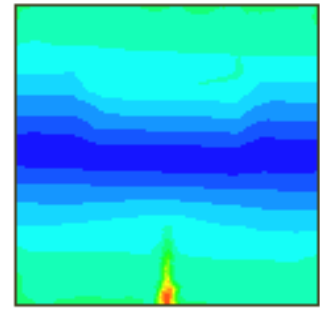

(b) $\mathrm{t}=\mathrm{T} / 4$

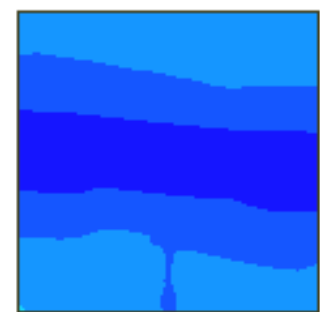

(d) $t=3 T / 4$

Figure 4. Field distribution on graphene surface

When the patch is excited, electrons are injected into the upper substrate (glass). This creates a Surface Plasma Polariton (SPP) between the graphene layer and the dielectric interface which are propagating along the antenna axis. This creates TM10 mode which is linearly distributed on the surface of the graphene layer as shown in Fig. 4.

The resonant frequency due to the generation of SPP waves is shown in Fig.5 and is compared with conventional nano-antenna with the same dimension as that of the proposed model. 


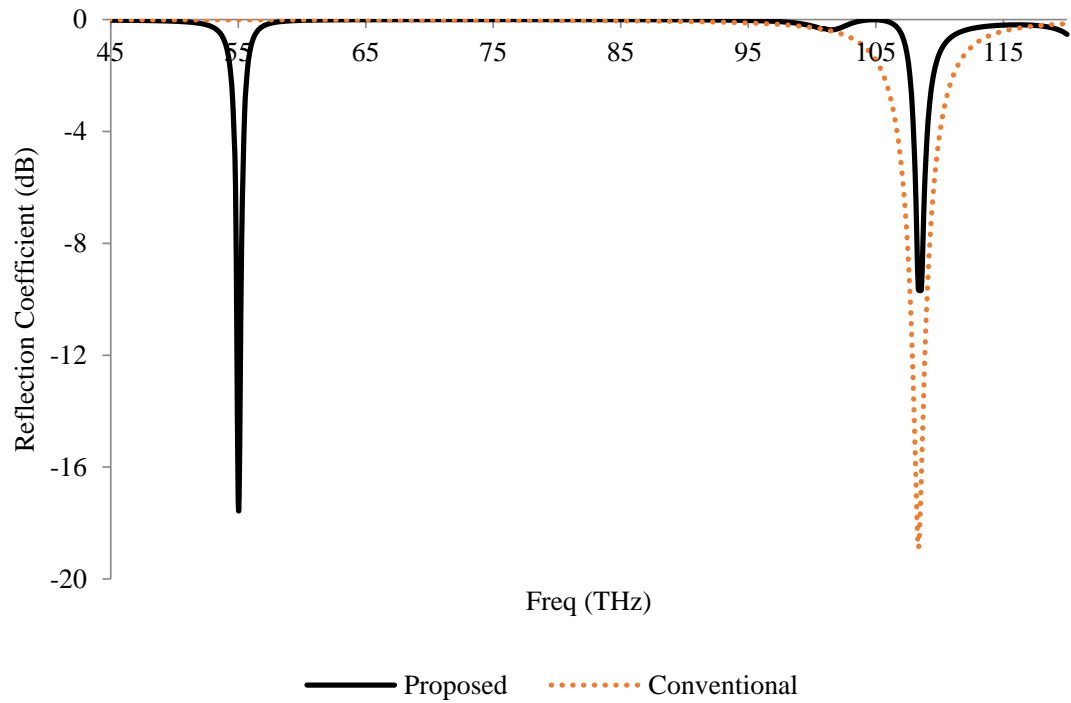

Figure 5. Comparison of operating frequencies between the proposed models with conventional nano-antenna.

It is observed in Fig. 5, conventional antenna resonates at $110 \mathrm{THz}$. Though it offers good bandwidth, it suffers from high propagation loss. This results in the poor coverage area of micrometer distance and also requires huge power requirement compared to poor requirement needed for the remaining circuits in the nano-devices. This puts difficulty in using conventional design in nano-antennas. This can be overcome by exploiting Surface Plasma Polariton (SPP) waves in combination with conventional nano-antenna as discussed above.
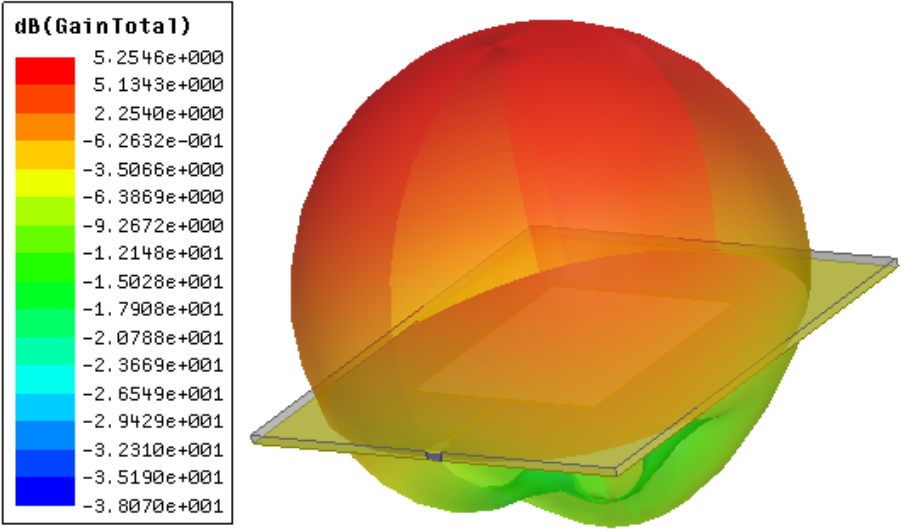

(a) 

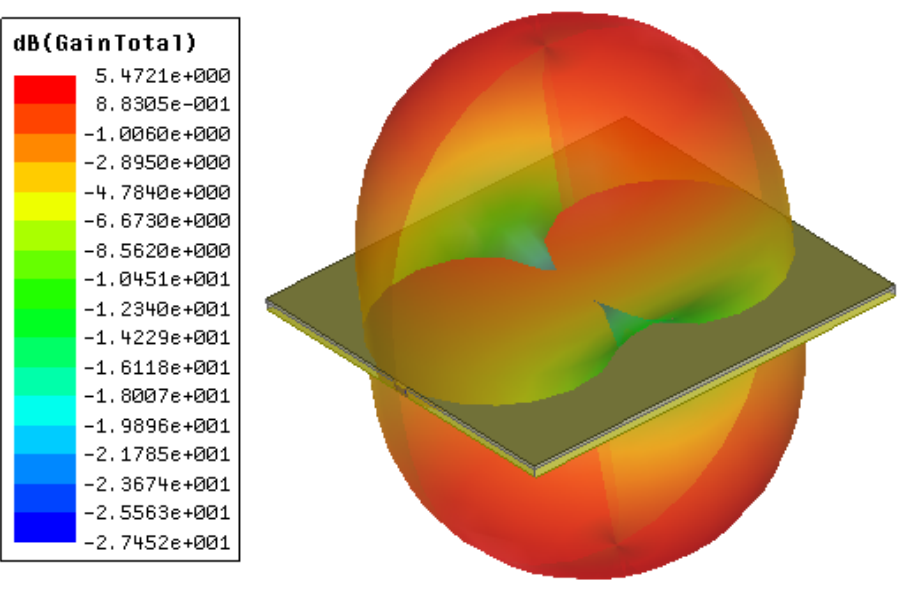

(b)

Figure 6. Gain Plot (a) Conventional Patch Antenna (b) Proposed Antenna

Figure 6 shows gain plot for conventional patch antenna and proposed antenna. It is observed that the conventional patch gives symmetrical unidirectional radiation pattern towards zenith with a peak gain of $5.25 \mathrm{~dB}$, whereas proposed antenna gives bi-directional radiation pattern with a peak gain of $5.47 \mathrm{~dB}$. This is due to presence of SPP waves which gives rise to additional radiation components towards ground plane region.

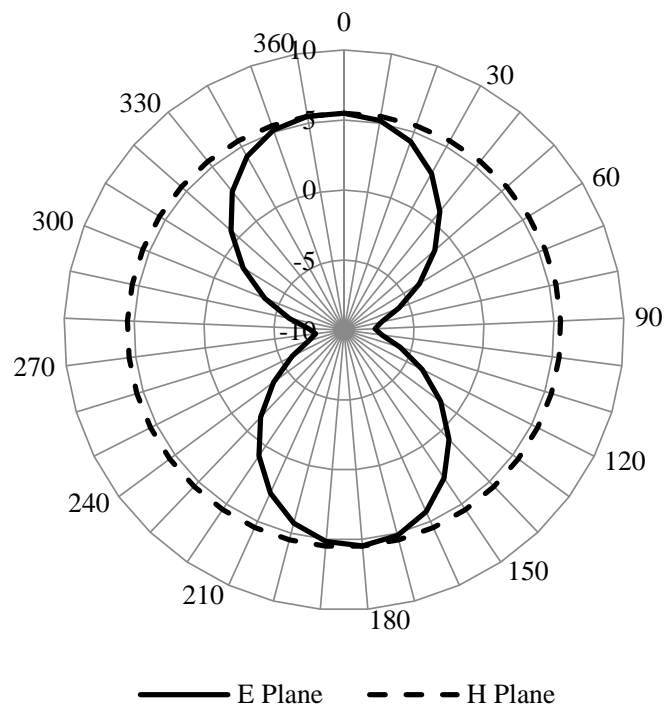

(a) 


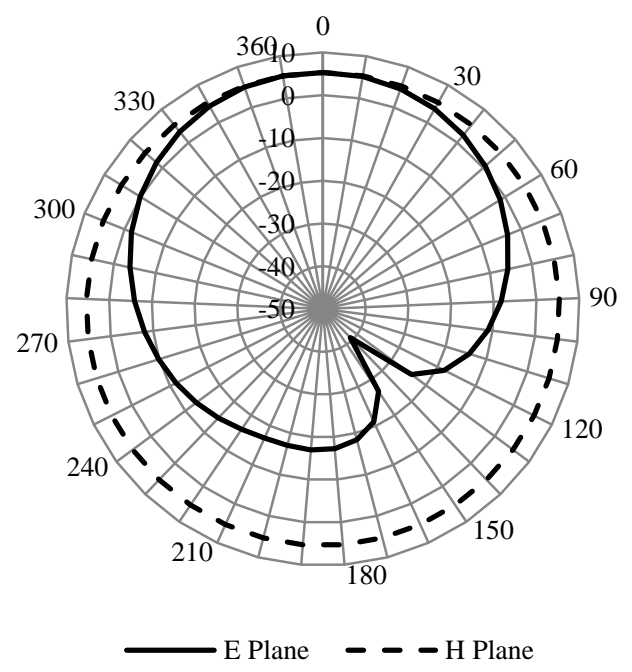

(b)

Figure 7. Radiation Pattern. (a) Graphene based nano-antenna. (b) Conventional nano-antenna.

The SPP waves generated propagates at a lower speed in the dielectric medium (glass) [6] and hence virtually increases electrical length when compared to its physical length. This makes the proposed model to resonate at $55 \mathrm{THz}$ near infrared region without sacrificing its performance characteristics and whereas the conventional model having same length and width as that of proposed model resonates at $110 \mathrm{THz}$ which is two times greater than the proposed model operating frequency and suffers from greater propagation loss. This makes graphene-based nano-antenna to cover two times the coverage distance with half the power requirement needed by the conventional nano-antenna and thereby makes it more suitable for communication in nano-devices when compared to conventional nano-antennas. The radiation characteristics of conventional nano-antenna are compared with graphene-based nano-antenna as shown in Fig. 7. The conventional antenna gives symmetrical radiation pattern toward propagation direction with a peak gain of $5.25 \mathrm{~dB}$ at zenith. However, the proposed antenna model gives symmetrical bidirectional radiation pattern with a peak gain of $5.47 \mathrm{~dB}$.

Table 1. Performance Comparison of Proposed Model with Conventional patch antenna

\begin{tabular}{lcc}
\hline \multicolumn{1}{c}{ Parameter } & Conventional Patch & Proposed Model \\
\hline Size & 1800nm x 1800nm x 36nm & 1800nm x 1800nm x 36nm \\
Operating Frequency & $110 \mathrm{THz}$ & $55 \mathrm{THz}$ \\
Reflection Coefficient & $18.4 \mathrm{~dB}$ & $17.6 \mathrm{~dB}$ \\
Gain & $5.25 \mathrm{~dB}$ & $5.47 \mathrm{~dB}$ \\
Directivity & $5.14 \mathrm{~dB}$ (Unidirectional) & $5.38 \mathrm{~dB}$ (Bidirectional) \\
\hline
\end{tabular}


Table 1 shows performance comparison of proposed antenna model with conventional antenna model. It is observed that the proposed antenna design reduces the overall antenna profile by $50 \%$ with sacrificing antenna performances along with bidirectional radiation pattern when compared with traditional antenna model.

\section{Conclusion}

In this letter, a graphene-based nano-antenna for communication in nano-networks is modeled and analyzed. The proposed model exploits the principle of surface plasma polariton (SPP) waves for its miniaturization. The performances of the proposed nano-antenna are characterized using finite difference time domain method and are compared with conventional nano-antenna. It is observed that the nano-antenna achieves greater miniaturization having half the size of the conventional antenna without sacrificing its performances at its operating frequency and hence it is better suitable for nano-network communication devices.

\section{Acknowledgements}

The author thanks the Thirumalai Engineering College, Kanchipuram for providing laboratory facility to carry our research work.

\section{References}

[1] Abdelhadi Ennajih, et al., (2017) A New Dual Band Printed Metamaterial Antenna for RFID Reader Applications, International Journal of Electrical and Computer Engineering (IJECE), 7(6), 3507-3514.

[2] Doucha, et al., (2017) A Leaky Wave Antenna Design Based on Half-mode Substrate Integrated Waveguide Technology for X Band Applications, International Journal of Electrical and Computer Engineering (IJECE), 7(6), 3467-3474.

[3] Junsei Horikawa, et al., (2014) Evaluation of Nano-Slot Antenna for Mid-Infrared Detectors,” Infrared Physics \& Technology, 67, 21-24.

[4] I. Llatser, et al., (2012) Graphene-based nano-patch antenna for terahertz radiation, Photonics Nanostructures - Fundamentals Appl., 10(4), 353-358.

[5] Ian F. Akyildiz and Josep Miquel Jornet, (2010) Electromagnetic wireless nanosensor networks, Nano Communication Networks, 1, 3-19.

[6] Josep Miquel Jornet and Ian F. Akyildiz, (2013) Graphene - based Plasmonic Nano-Antenna for Terahertz Band Communication in Nanonetworks, IEEE Journal on Selected Areas in Communications, 31(12), 685-694.

[7] Abbasi, et. al., (2018) IEEE Access Special Section Editorial: Nano-Antennas, Nano-Transceivers and Nano-Networks/Communications, IEEE Access, 6, 8270-8272.

[8] L.Ju, , et al., (2011) Graphene plasmonics for tunable terahertz metamaterials, Nature Nanotechnol., 6, 630-634.

[9] Mona Nafari and josep miquel jornet, (2017) Modeling and Performance Analysis of Metallic Plasmonic Nano-Antenna for Wireless Optical 
Communication Nanonetworks, IEEE Access, 56389-6398.

[10] J. Dorfmüller, et al., (2010) Plasmonic nanowire antennas: Experiment, simulation, and theory, Nano Lett., 10(9), 3596-3603.

[11] Quzwain, et. al., (2015) Gain Enhancement of Octagon Microstrip Yagi Antenna Utilizing 1-D Photonic Crystal (PCs) Cover, Proceeding of International Conference on Electrical Engineering, Computer Science and Informatics (EECSI 2015), Palembang, Indonesia, 19 -20 August 2015, 194-198.

[12] Palacios, T., Hsu A. and Wang, H. (2010) Applications of graphene devices in RF communications, IEEE Communications Magazine, 48(6), 122-128.

[13] Renato Iovine, Richard Tarparelli and Lucio Vegni, (2014) Electromagnetic Analysis of Graphene Nanoparticles Operating in the TeraHertz Band, Advances in Nanoparticles, 3, 72-76.

[14] Bedir B. Yousif and Ahmed S. Samra, (2012) Modeling of Optical Nanoantennas, Physics Research International, Article ID 321075, 1-10.

[15] Seyed Arash Naghdehforushha and Gholamreza Moradi, (2018) Plasmonic patch antenna based on graphene with tunable terahertz band communications, Optik, Vol. 158, 617-622.

[16] G.Samanta and D.Mitra, (2018) Wideband THz antenna using graphene based tunable circular reactive impedance substrate, Optik, vol. 158, 1080-1087.

[17] Novotny, L. (2007) Effective wavelength scaling for optical antennas, Physical Review Letters, vol.98, 266802. 